\title{
Dynamic characteristics and co-occurrence patterns of microbial community in tobacco leaves during the 24-month aging process
}

\author{
Jiaxi Zhou', Lifei Yu', Jian Zhang ${ }^{1}$, Jing Liu ${ }^{2}$ and Xiao Zou ${ }^{1 *}$ (D)
}

\begin{abstract}
Purpose: Microorganisms are important in tobacco aging. These are used to improve the quality of tobacco leaves after threshing and redrying. However, the response of microbial community to the storage environment and time during the tobacco aging process has been less explored. This study aimed to characterize the dynamic changes in microbial community composition and diversity in tobacco leaf samples.

Methods: In this study, $16 \mathrm{~S}$ and ITS rRNA gene amplicon sequencing techniques were used to characterize the composition, diversity, and co-occurrence of the microbial community in tobacco leaves stored in two different cities during the 24-month aging. Furthermore, the activities of several enzymes were measured spectrophotometrically, and the correlation between the microbiota and enzyme activity was analyzed by network analysis.

Results: Shannon diversity and Chao richness of bacterial communities gradually increased during the first 18 months, whereas those of the fungal community decreased. The relative abundance of Proteobacteria decreased, whereas that of Actinobacteria and Bacteroidetes increased. The proportion of Ascomycota gradually increased during the first 18 months and then rapidly decreased, whereas the proportion of Basidiomycota exhibited a completely opposite pattern. The change in the composition of bacterial community and dominant genera in leaves was not significant between Guiyang city and Maotai city storerooms, but that in the fungal community was significant. The network analysis revealed that fungal networks were more complex and compact than bacterial networks, and a strong negative correlation existed between bacteria and fungi. Moreover, the bacterial microbiome showed a strong positive association with amylase activity, while the fungal microbiome positively correlated with cellulase activity.

Conclusions: This study demonstrated a significant spatiotemporal heterogeneity in the composition of the microbial community during tobacco aging and highlighted the possible influence of the interactions and enzyme activity on microbial diversity and composition. The findings provided a scientific basis for using microorganisms to regulate and control tobacco aging.
\end{abstract}

Keywords: Co-occurrence patterns, Enzymatic activity, Microbial community diversity, Network analysis, Tobacco aging

\footnotetext{
* Correspondence: xzou@gzu.edu.cn

${ }^{1}$ Institute of Fungal Resources, Department of Ecology, College of Life

Sciences, Guizhou University, Guiyang, China

Full list of author information is available at the end of the article
}

(c) The Author(s). 2021 Open Access This article is licensed under a Creative Commons Attribution 4.0 International License, which permits use, sharing, adaptation, distribution and reproduction in any medium or format, as long as you give appropriate credit to the original author(s) and the source, provide a link to the Creative Commons licence, and indicate if changes were made. The images or other third party material in this article are included in the article's Creative Commons licence, unless indicated otherwise in a credit line to the material. If material is not included in the article's Creative Commons licence and your intended use is not permitted by statutory regulation or exceeds the permitted use, you will need to obtain permission directly from the copyright holder. To view a copy of this licence, visit http://creativecommons.org/licenses/by/4.0/. 


\section{Introduction}

Flue-cured tobacco is an important non-edible economic crop, but unaged tobacco is not suitable for making cigarette products directly because it has a sharp, disagreeable odor and an undesirable aroma, and produces irritating smoke (Zhao et al. 2007). The aging process takes approximately 2 years and is typically used to improve the quality of flue-cured tobacco because it can greatly improve the aroma and color, reduce smoke irritation, and improve the overall tobacco quality (Su et al. 2011). Increasing evidence shows that microorganisms are crucial in the aging process. Microbial metabolism can promote the transformation of proteins, starch, cellulose, and other macromolecules in the tobacco leaf substrate, thus increasing the contents of aromatic compounds such as ionone (Maldonado-Robledo et al. 2003) and reducing the contents of harmful components such as nicotine and tobacco-specific nitrosamines (Liu et al. 2015; Vigliotta et al. 2007).

Modern molecular biology techniques can be used to improve the understanding of tobacco-associated microbial community structure and function. Microbial community diversity has been investigated in tobacco and tobacco products using culture-independent molecular biology techniques, such as denaturing gradient gel electrophoresis (Zhao et al. 2007), 16S rRNA clone libraries (Su et al. 2011), restriction fragment length polymorphisms (Huang et al. 2010), and Illumina MiSeq sequencing (Law et al. 2016). Sphingomonas, Bacillus, Pseudomonas, Lactococcus, Stenotrophomonas, Acinetobacter, Methylobacterium, Erwinia, and Pantoea were identified as the predominant bacterial populations; and Neophaeosphaeria, Cladosporium, Lodderomyces, Tilletiopsis, Symmetrospora, Xeromyces, Wallemia, Rhodotorula, Cercospora, Alternaria, Cryptococcus, and Aspergillus were identified as the prevalent fungal groups (Villemur et al. 2009; Ye et al. 2017; Wang et al. 2018; Zhang et al. 2020; Zhou et al. 2020).

Alternatively, the structure and function of microbial communities are usually dynamic and affected by environmental conditions (Kinkel 1997). Storage conditions also significantly affect the composition of tobacco microbial communities (Larsson et al. 2008). During the tobacco aging process, tobacco leaves (e.g., sources and varieties) and storage conditions (e.g., temperature and humidity) provide a variety of niches for microbial growth and reproduction, and these factors determine the tobacco microbe species, abundance, and evolution (Chopyk et al. 2017). Therefore, tobacco companies usually transfer local tobacco leaves to other cities for aging. However, the response of microbial communities to different storage environments remains unclear.

Microorganisms are the main driving force behind the circulation and transformation of the tobacco leaves through the whole process of aging. However, a dry environment is a necessary prerequisite for tobacco fermentation or aging. In industrial production, the moisture content of tobacco leaves should not exceed $13 \%$ and the relative humidity of the environment should not exceed 70\% during the aging process (Lian et al. 1998), restricting the growth of most microorganisms. Furthermore, the relationships between microbial taxa also shape the microbial community structure (Barberán et al. 2012). The network analysis of significant taxon co-occurrence patterns can help reveal the structure of complex microbial communities across spatial or temporal gradients. Recently, the network analysis has been widely applied to reveal ecological linkages among microorganisms in complex ecosystems, such as in biogas digesters (Rui et al. 2015), sludge (Zhao et al. 2014), plants (Cao et al. 2020), soil (Shetty et al. 2017), and humans (Sun et al. 2011). However, the existence of direct or indirect interactions among microbial taxa in tobacco leaves during aging remains unclear. This study characterized the dynamic changes in microbial community composition and diversity in tobacco leaf samples collected from five different periods in Guiyang city (GY) and Maotai city (MT) for 2 years by employing high-throughput sequencing technology. Additionally, the network analysis was also used to reveal the correlation among microbial taxa and enzyme activities that coexisted in tobacco leaves during the aging process.

\section{Materials and methods \\ Sample collection}

Tobacco leaves were sampled from a warehouse located in GY and MT, Guizhou Province, China, and collected by China Tobacco Guizhou Industry Co., Ltd. (Guiyang, China) between July 2015 and July 2017. Guiyang is about $1100 \mathrm{~m}$ above sea level, with a monthly mean relative humidity ranged from 70.0 to $89.2 \%$, and a monthly mean temperature of $24.3^{\circ} \mathrm{C}$ in summer and $4.1^{\circ} \mathrm{C}$ in winter. Maotai is about $400 \mathrm{~m}$ above sea level, with a monthly mean relative humidity ranged from 67.3 to $84.8 \%$, and a monthly mean temperature of $27.2^{\circ} \mathrm{C}$ in summer and $5.4{ }^{\circ} \mathrm{C}$ in winter.

The grade of all samples was C3F (the third-grade tobacco leaves with yellow-orange color in the middle of the plant), and all tobacco plants were harvested from Baoshan, Yunnan Province, China, in 2014. In total, 200 $\mathrm{kg}$ tobacco leaves were aged for 24 months in a tobacco carton (1080-mm long $\times$ 650-mm wide $\times 685-\mathrm{mm}$ high), maintained at room temperature $\left(6-32^{\circ} \mathrm{C}\right)$, and sampled after $0,6,12,18$, and 24 months. During each of the five sampling periods, $1.5 \mathrm{~kg}$ tobacco leaves were arbitrarily collected from $15 \mathrm{~cm}$ below the top of the cartons by the five-point method. A total of 10 samples were obtained as follows: 0 months (GY-0 and MT-0), 6 months (GY-6 and MT-6), 12 months (GY-12 and MT-12), 18 months 
(GY-18 and MT-18), and 24 months (GY-24 and MT24). All samples were packed in sterile plastic bags and transported to the Institute of Fungal Resources at Guizhou University for storage at $-20^{\circ} \mathrm{C}$. In the following experiment, $500 \mathrm{~g}$ tobacco leaves were weighed and ground with liquid nitrogen; then, $100 \mathrm{~g}$ ground tobacco leaves were weighed for DNA extraction, and the rest were tested for enzyme activity.

\section{Determination of enzymatic activity in tobacco leaves during the aging process}

According to the color reaction between the degradation products (glucose, maltose, and galacturonic acid) and 3, 5-dinitrosalicylic acid (DNS), the activities of amylase, cellulose, and pectinase were measured spectrophotometrically (Malhotra et al. 2000; Wang et al. 2008; Su et al. 2016). Each sample was repeated three times. Independent sample $t$ test and one-way analysis of variance (ANOVA) were employed to assess the statistical significance of enzymatic activities under different spatiotemporal conditions using SPSS Statistics version 19 (IBM, IL, USA). In all cases, one unit of enzyme activity was defined as the amount of enzyme releasing $1 \mu \mathrm{mol}$ of reducing sugar under the assay conditions (Minjares-Carranco et al. 1997). The enzyme activity was expressed in units of $\mathrm{U} \mathrm{g}^{-1}$.

\section{DNA extraction and MiSeq sequencing}

The microbial genomic DNA was extracted using the E.Z.N.A. soil kit (Omega Biotek, GA, USA), and the DNA concentration and purity were monitored by electrophoresis on $1 \%(\mathrm{w} / \mathrm{v})$ agarose gels. The primers $338 \mathrm{~F}$ (5' -ACTCCTACGGGAGGCAGCAG-3') and 806R (5' GGACTACHVGGGTWTCTAAT-3') were used to amplify the V3-4 variable region of the bacterial $16 \mathrm{~S}$ rRNA gene (Xu et al. 2016). The fungal ITS gene was amplified with the primers ITS1F $\left(5^{\prime}\right.$-CTTGGTCATT TAGAGGAAGTAA-3') and ITS2R (5'-GCTGCGTTCT TCATCGATGC-3') (Adams et al. 2013). The amplification products were sequenced using the Illumina HiSeq2500 PE250 platform (Majorbio Bio-pharm Technology Co., Ltd., Shanghai, China). Libraries were constructed according to the TruSeq DNA Sample Prep Kit protocol (Illumina, CA, USA). All Illumina HiSeq sequencing data were deposited into the NCBI Sequence Read Archive database under BioProject accession number PRJNA513397, https://www.ncbi.nlm.nih.gov/ bioproject/?term=PRJNA513397.

\section{Data analysis}

Raw reads from original DNA fragments were merged and quality-filtered using FLASH version 1.2.7 (Magoč and Salzberg 2011) and Trimmomatic version 0.32. The UPARSE version 7.1 was used for chimera removal, and operational taxonomic units (OTUs) were clustered with $a \geq 97 \%$ similarity (Edgar 2013). Bacterial gene sequences were annotated with taxonomic information using the RDP classifier against the SILVA $13216 \mathrm{~S}$ rRNA database (Rognes et al. 2016). The OTU taxonomic information of fungi was obtained by aligning each representative sequence against the Unit 8.0 ITS database (Kõljalg et al. 2013). The standard sequence number was compared with that of the sample with the least number of sequences to normalize the OTU abundance. Alpha diversities of the bacteria and fungi, including Chao and Shannon values, were calculated at the OTU level using QIIME. Beta diversity analysis was performed based on the Bray-Curtis distance of the relative abundance of OTUs in nonmetric multidimensional scaling (NMDS). Analysis of similarity (ANOSIM) was applied to test the differences in the changes in bacterial and fungal community compositions across two sites. These data were analyzed on the free online platform of Majorbio Cloud Platform (www.majorbio.com).

\section{Network analysis}

For network analysis, the OTUs were grouped at the genus level, and only those genera with more than 10 sequences in at least three samples were used in the following analysis. The correlation between the microbial communities (bacterial and fungal genera) and enzyme activities was examined by calculating all possible pairwise Spearman's rank correlations using SPSS 19. A valid interaction event was considered to be a robust correlation if the Spearman's correlation coefficient $(r)$ was $>0.6$ or $<-0.6$ and statistically significant $(P<0.05)$ (Zhao et al. 2014; Rui et al. 2015). The correlation matrix constructed by Spearman's correlations was imported into Cytoscape version 3.5.1 to explore and visualize the network (Shannon et al. 2003). In the correlation network, each node represented one genus or an enzyme, and each edge represented a strong and significant correlation (Zhao et al. 2014). A set of the network topological parameters, such as the degree, closeness centrality (CC), and betweenness centrality (BC), were calculated using the Cytoscape plugin NetworkAnalyzer (Assenov et al. 2008). Furthermore, the keystone taxa for the bacterial and fungal network (Sporns et al. 2007; Gonzalez et al. 2010) were explored based on high centrality (BC and $\mathrm{CC}$ ) scores; the supergeneralist of the network and a candidate for a hub of the network was the node with higher $\mathrm{BC}$ and $\mathrm{CC}$ values (Zhao et al. 2019b).

\section{Results}

Analysis of the enzyme activity

Starch, cellulose, and pectin worked as the important components of tobacco leaves, so its degradation process 
was closely related to the activities of enzymes. The independent sample $t$ test showed no significant difference in enzyme activities between GY and MT storerooms, but one-way ANOVA showed that enzyme activities changed significantly with aging time (Fig. 1). The amylase activity increased significantly at first and then gradually decreased, and the maximum value appeared after 12 and 18 months in MT $\left(6.74 \mathrm{Ug}^{-1}\right)$ and GY $(7.95 \mathrm{U}$ $\left.\mathrm{g}^{-1}\right)$ storerooms, respectively. The pectinase activity in the later 12 months was significantly higher than that in the first 6 months, with the highest activity after 12 months $\left[5.76 \mathrm{U} \mathrm{g}^{-1}(\mathrm{GY})\right.$ and $5.56 \mathrm{U} \mathrm{g}^{-1}(\mathrm{MT})$, respectively]. In contrast, the cellulase activity decreased significantly and was the lowest after 12 months $\left(5.76 \mathrm{U} \mathrm{g}^{-1}\right)$ in the GY storeroom.

\section{Diversity of bacterial and fungal communities}

A total of 429,490 bacterial and 565,872 fungal sequences were obtained and clustered into 1586 and 885 OTUs, respectively, from 10 tobacco leaf samples collected in 5 periods during the aging process. As shown in Fig. 2 a and b, the Shannon and Chao indices of bacteria increased from 3.93 and 391.63 to 5.39 and 839.39 during the first 18 months, respectively, in the GY storeroom, but decreased to 4.86 and 724.26 by 24 months. In the MT storeroom, Shannon's index of bacteria fluctuated from 5.11 to 5.26 . However, the Chao index was the highest after 24 months (651.21) and the lowest at the beginning (355.00). The fungal diversity and richness in the GY storeroom decreased significantly from 3.15 and 451.29 to 1.91 and 284.69 during the first 6 months, respectively, and reached the lowest after 18 months (1.38 and 232.14, respectively). In the MT storeroom, the fungal diversity increased from 2.38 to 2.63 in the first 6 months, then decreased to 0.33 after 18 months, and finally recovered to 1.97 after 24 months (Fig. 3 a and $b$ ). However, the richness dropped significantly from 417.84 to 12.5 in the first 18 months before recovering to 144.05. In general, the Shannon and Chao indices of the fungi in the MT storeroom were lower than those in the GY storeroom, but the difference was not significant. The aforementioned results indicated that bacteria and fungi worked together in the early stage of tobacco aging, while the latter was dominated by bacteria.

\section{Bacterial and fungal community composition}

Analyses of NMDS based on Bray-Curtis distance demonstrated that the bacterial and fungal communities in GY and MT storerooms could be divided into two groups (Fig. 4). Furthermore, ANOSIM analysis demonstrated significant differences in the changes of fungal community composition between the two storage rooms $(P=0.017<0.05)$, but no statistically significant differences were identified for bacterial communities $(P=$ $0.110>0.05$ ).

Proteobacteria, Firmicutes, Actinobacteria, Bacteroidetes, Acidobacteria, Chloroflexi, Deinococcus-Thermus, and Patescibacteria were the main bacterial groups in the tobacco aging system, and the dominant bacterial phyla were Proteobacteria (43.65-91.14\%) and Firmicutes (2.84-23.96\%) based on the average relative abundance. Obviously, the proportion of Proteobacteria gradually decreased during the aging process, and its average relative abundance was higher in the GY storeroom than in the MT storeroom. The relative abundance of Proteobacteria decreased from $91.14 \%$ at the beginning to $66.14 \%$ by 18 months in the GY storeroom, and then slightly recovered to $71.61 \%$ by 24 months. Similarly, the relative abundance of Proteobacteria decreased from $65.21 \%$ after 6 months to $43.65 \%$ after 18

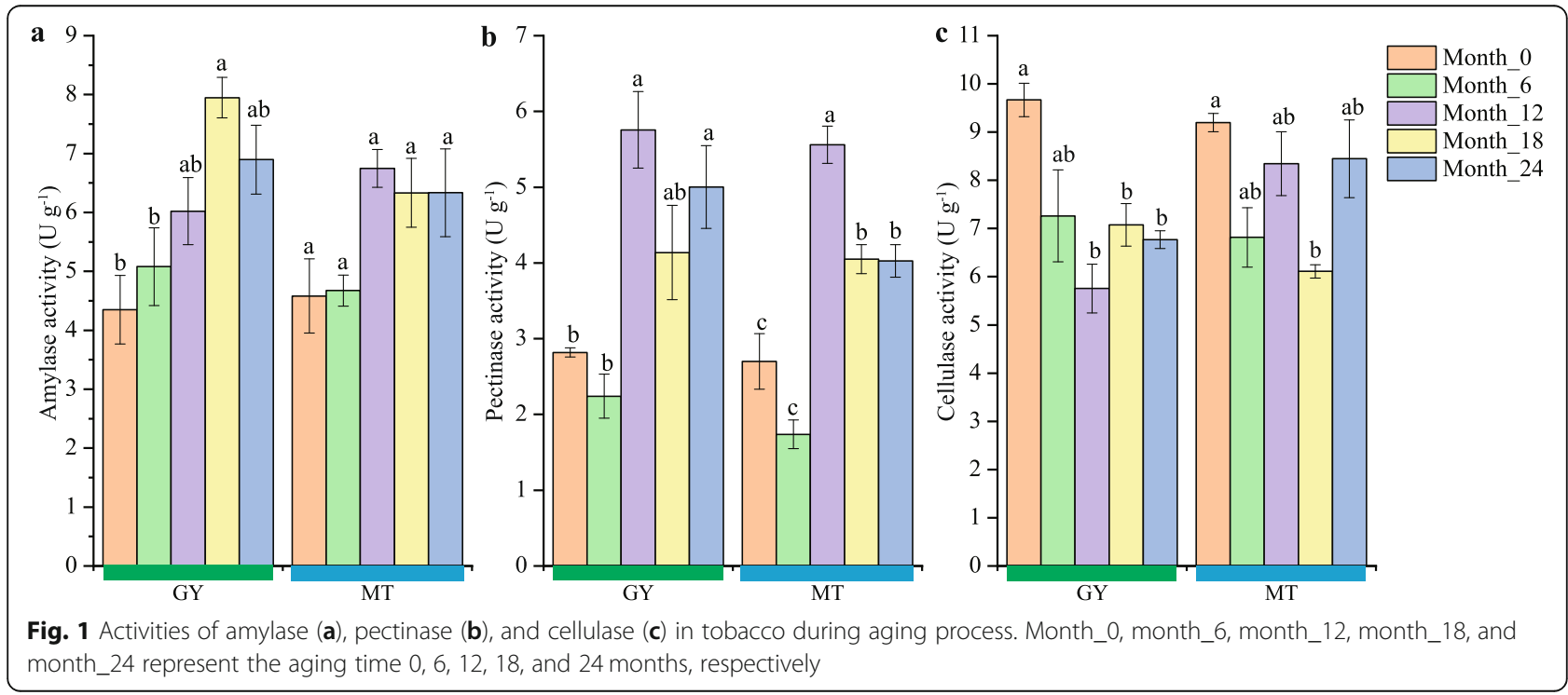




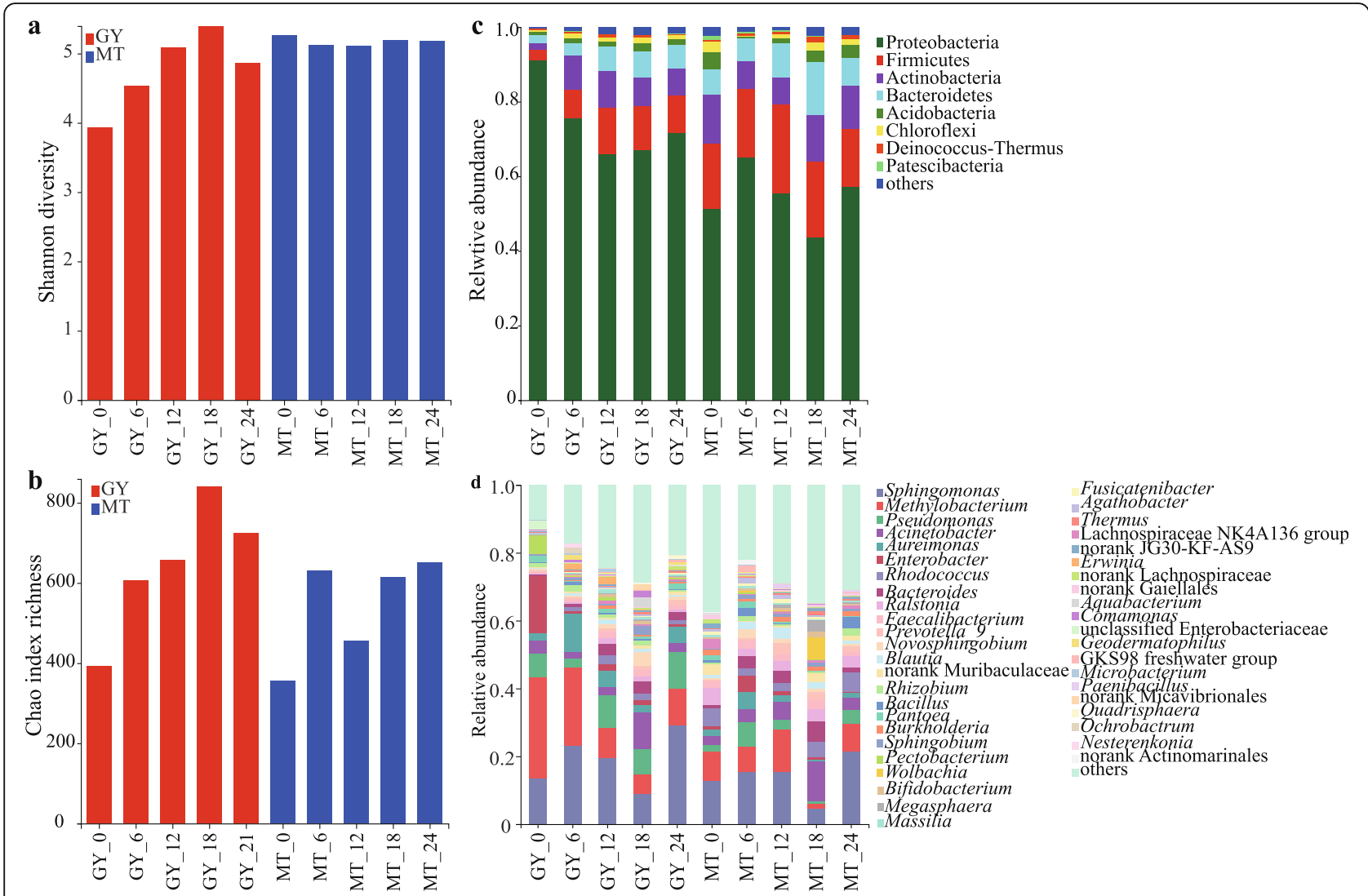

Fig. 2 Diversity and composition of bacterial communities in tobacco leaves during the 24-month aging process. a Shannon diversity and $\mathbf{b}$ Chao richness values of bacterial communities; c bacterial community composition at the phylum level (phyla with a relative abundance of less than 0.01 were assigned to "other"); and $\mathbf{d}$ bacterial community composition at the genus level (genus with a relative abundance of less than 0.01 was assigned to "other")

months in the MT storeroom and then recovered to $57.17 \%$ by 24 months (Fig. 2c). Contrary to Proteobacteria, Firmicutes, Actinobacteria, and Bacteroidetes gradually increased in relative abundance, and their average abundance in the MT storeroom (34.03-47.06\%) was higher than that in the GY storeroom (6.73-28.71\%). A total of 317 bacterial genera were detected, of which 44 taxonomic groups had a relative abundance greater than 1\% (Fig. 2d). Sphingomonas and Methylobacterium were the most dominant genera. The relative abundance of Sphingomonas after $0,6,12,18$, and 24 months of tobacco leaf aging was 13.46\%, 23.14\%, 19.58\%, 8.86\%, and $29.05 \%$, respectively, in the GY storeroom, and $12.69 \%$, $15.65 \%, 15.43 \%, 4.54 \%$, and $21.50 \%$, respectively, in the MT storeroom. The relative abundance of Methylobacterium decreased gradually from $29.98 \%$ after 0 months to $5.91 \%$ after 18 months in the GY storeroom, and from $12.64 \%$ after 12 months to $1.42 \%$ after 18 months in the MT storeroom. In addition, the abundance of Enterobacter decreased from $13.68 \%$ and $4.765 \%$ to $0.71 \%$ and $0.38 \%$ throughout the entire aging process in GY and MT storerooms, respectively. The relative abundance of Acinetobacter showed a consistent trend between GY and MT storerooms, first increasing and then decreasing, with the highest after 18 months $(10.61 \%$ and $11.98 \%$, respectively).

A total of 6 phyla and 389 genera of fungi were detected from 10 tobacco leaf samples. The dominant fungal phyla were Basidiomycota and Ascomycota in the GY storeroom. The relative abundance of Basidiomycota decreased significantly from $68.08 \%$ after 6 months to $15.06 \%$ after 18 months and then recovered rapidly to $77.28 \%$ after 24 months. In contrast, the abundance of Ascomycetes increased significantly from $28.97 \%$ after 6 months to $84.55 \%$ after 18 months and then decreased to $22.06 \%$ after 24 months (Fig. 3c). In the MT storeroom, Ascomycota was the most dominant phylum; its relative abundance gradually increased after treatment, reaching the maximum (more than 99\%) after 18 months. In addition, after 18 months, the proportion of Basidiomycota also increased (18.74\% after 24 months) with a decrease in the abundance of Ascomycota. At the genus level, 18 taxonomic groups had a relative abundance greater than $1 \%$ for all samples (Fig. 3d). The dominant genera in different storerooms were different. In the GY storeroom, Sampaiozyma was the most 


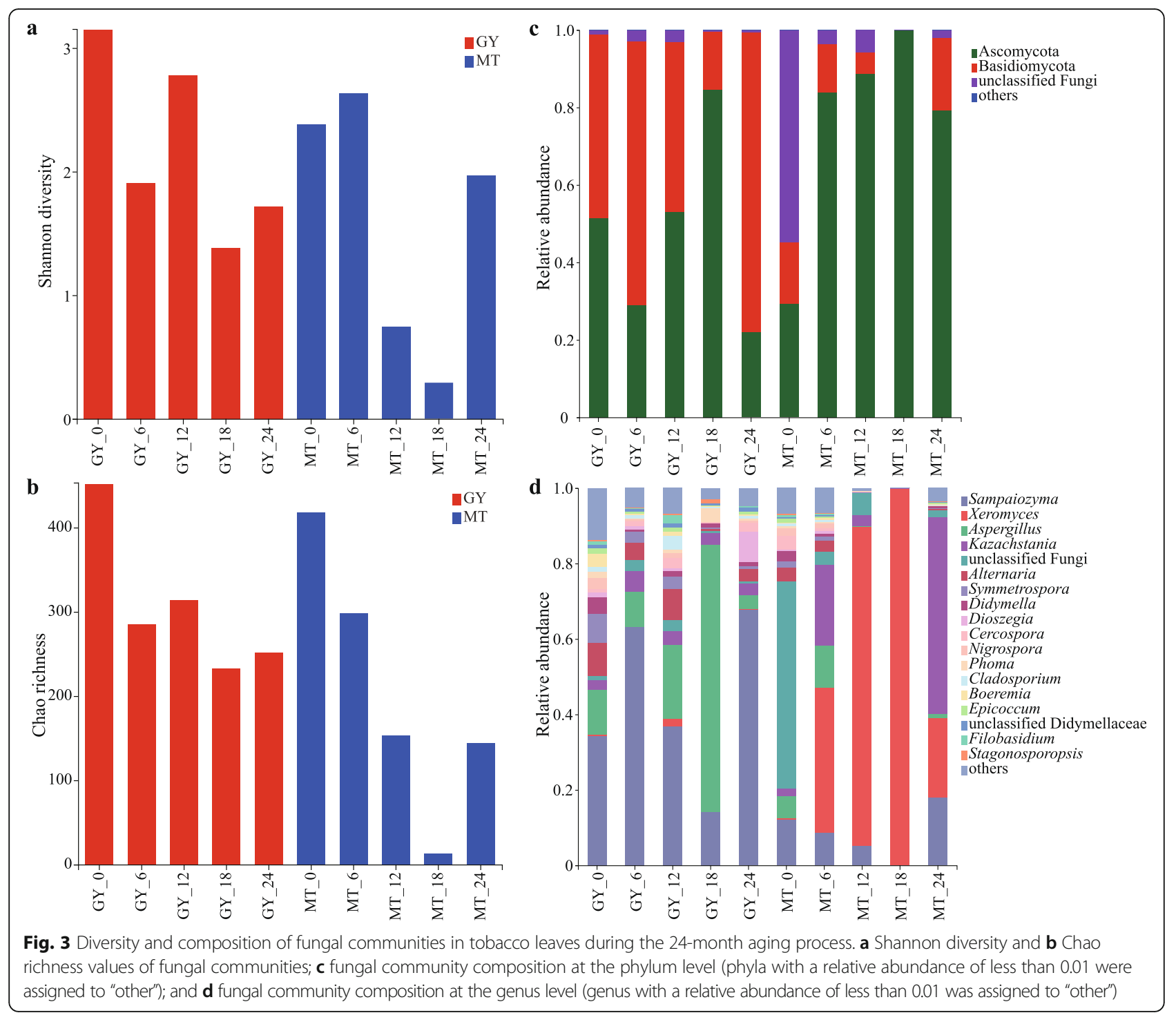

dominant genus in the whole aging process except Aspergillus after 18 months. However, Xeromyces had a distinct advantage in the MT storeroom ranging from 6 to 18 months. In addition, Fig. $3 \mathrm{~d}$ shows that the abundance of most genera of Ascomycota, such as Alternaria and Cladosporium, gradually decreased during the aging process in the two storerooms, whereas the abundance of Dioszegia belonging to Basidiomycota gradually increased in the GY storeroom.

\section{Co-occurrence among bacterial and fungal taxa}

The bacterial community co-occurrence patterns during the aging process were inferred based on strong and significant correlations $(P<0.05, r>0.6)$. A total of 97 strong positive correlations and 24 strong negative correlations were identified from 46 bacterial genera (Fig. 5a). The average network path length was 2.914 edges with a diameter of six edges; the network density and clustering coefficient were 0.116 and 0.438 , respectively. The cooccurring taxa were mainly distributed in Proteobacteria (39.13\%, ratio of targeted nodes to total nodes), Firmicutes (36.99\%), Bacteroidetes (10.87\%), Actinobacteria (8.70\%), Acidobacteria (2.17\%), and Deinococcus-Thermus (2.17\%). The top five genera (Burkholderia, Blautia, Fusicatenibacter, Rhodococcus, and Methylobacterium) were identified based on high centrality (BC and CC) scores. Taxa that individually or in a guild exerted a considerable influence on microbiome structure and functioning, irrespective of their abundance across space and time, were highly connected (Berry and Widder 2014). Furthermore, genera from different phyla (interphylum) had high cooccurrence $(69.17 \%$, ratio of targeted edges to total edges). The incidence of positive occurrence was the highest between the members of Firmicutes and Proteobacteria (up to $18.33 \%$ ), such as between Megamonas and Ralstonia, between Blautia and Aquabacterium, Novosphingobium, 

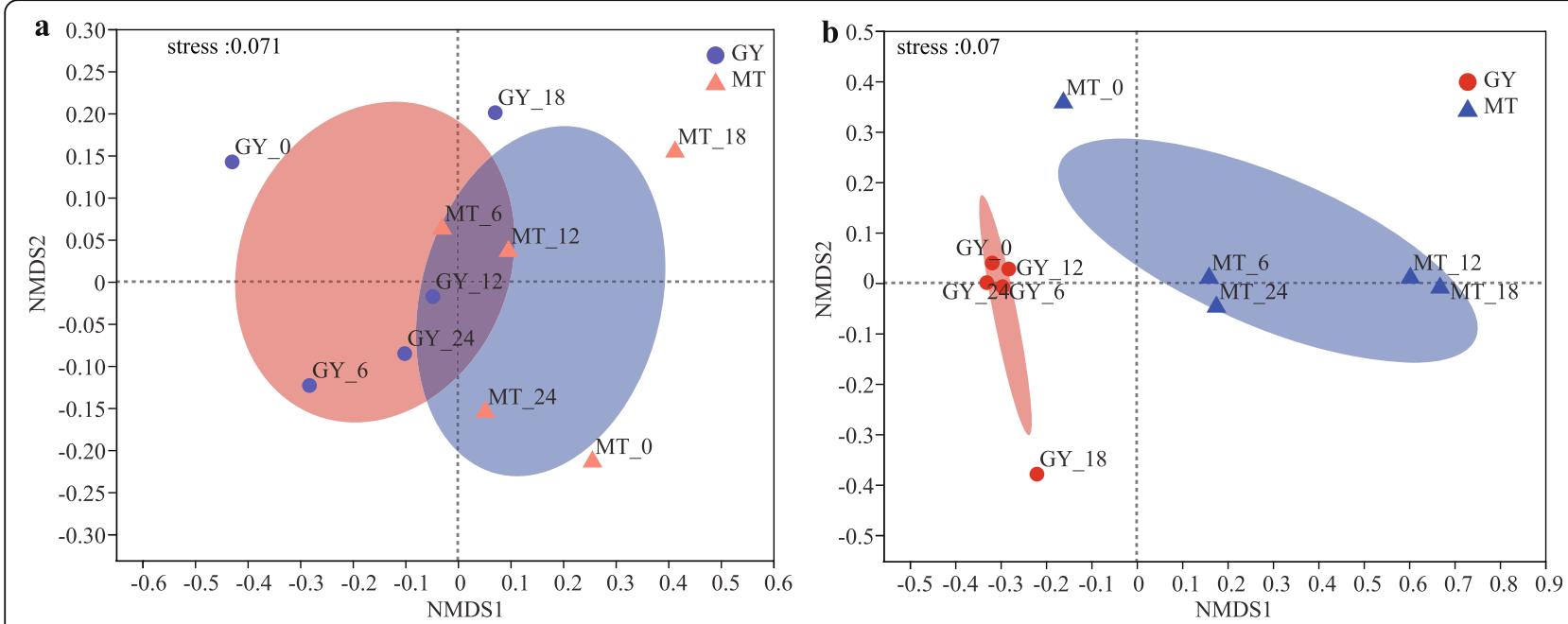

Fig. 4 NMDS analysis of bacterial (a) and fungal (b) community in tobacco aging at OTU level

and Sphingobium. However, the incidence of negative occurrence was the highest between Proteobacteria and Actinobacteria (up to 5.00\%), such as between Burkholderia and Aureimonas, Pectobacterium, and Stenotrophomonas. For intraphylum correlation, Firmicutes had a high incidence of positive occurrence (10.83\%), whereas Proteobacteria $(7.50 \%)$ had a higher incidence of the negative occurrence. In addition, Methylobacterium and members of other genera had a high negative incidence (5.00\%). For example, negative correlations were found between Methylobacterium and Lactobacillus, Bifidobacterium, Rhodococcus, Bacteroides, Novosphingobium, Faecalibacterium, and Thermus.

The correlation network analysis of fungi identified 552 strong positive correlations and 4 strong negative correlations from 488 fungal genera $(P<0.05, r>0.6$; Fig. $5 \mathrm{~b})$. The average network path length was 1.678 edges with a diameter of five edges. The fungal network had a higher network density and clustering coefficient (0.493 and 0.740, respectively) compared with the bacterial network, indicating that the fungal microbiome associations were more tightened than the bacterial microbiome associations. The cooccurring taxa were mainly distributed in Ascomycota (82.61\%) and Basidiomycota (19.42\%). According to the combined score of high CC and high BC, the top five genera identified were Nigrospora, Boeremia, Alternaria, Cladosporium, and Corynespora. They played critical roles as keystone taxa in the tobacco during the aging process. Furthermore, the incidence of co-occurrence within a phylum (intraphylum) was the highest among genera from Ascomycota (65.83\%). In the fungal network, Xeromyces showed significant and strong negative correlations with other genera, such as Neoascochyta, Microdiplodia, and Phoma.

\section{Co-occurrence between bacterial and fungal taxa}

The co-occurrence network of bacterial and fungal microbiomes was constructed to understand the microbial cross-kingdom associations. A total of 376 pairs of significant and strong correlations were identified from 90 genera taxa, including 45 bacterial genera and 45 fungal genera (Fig. 6a). The incidence of negative occurrence was higher than the incidence of positive occurrence in the bacteria-fungus network; they accounted for $95.48 \%$ (ratio of targeted edges to total edges) and 4.52\%, respectively. For example, Megamons, Burkholderia, Prevotella_9, Collinsella, and Cutibacterium showed a negative correlation with Mycosphaerella, Golubevia, Immersiella, and Plectosphaerella. Pectobacterium positively correlated with Boeremia and Malassezia, Bacillus with Kazachstania and Sordaria, and Methylobacterium with Fusarium. Overall, these results indicated that the most bacteria-fungi interactions might be mutually exclusive during tobacco aging.

\section{Relationship between the microbial microbiome and enzyme activities}

The co-occurrence network of amylase, cellulase, and pectinase with bacterial and fungal microbiomes was constructed to further characterize the effects of enzyme activity on the dynamics of microbiomes in tobacco leaves during the aging process. In the microbiome-enzyme network, 54 strong positive correlations and 21 strong negative correlations were identified between 75 taxa and 3 enzyme activities (Fig. 6b). Not surprisingly, $80 \%$ of the taxa were closely related to the activity of amylase, with positive correlations for bacteria and negative correlations for fungi. Starch, accounting for 2-6\% of the total biomass of tobacco leaves, was an important carbon source for microbial growth and reproduction 


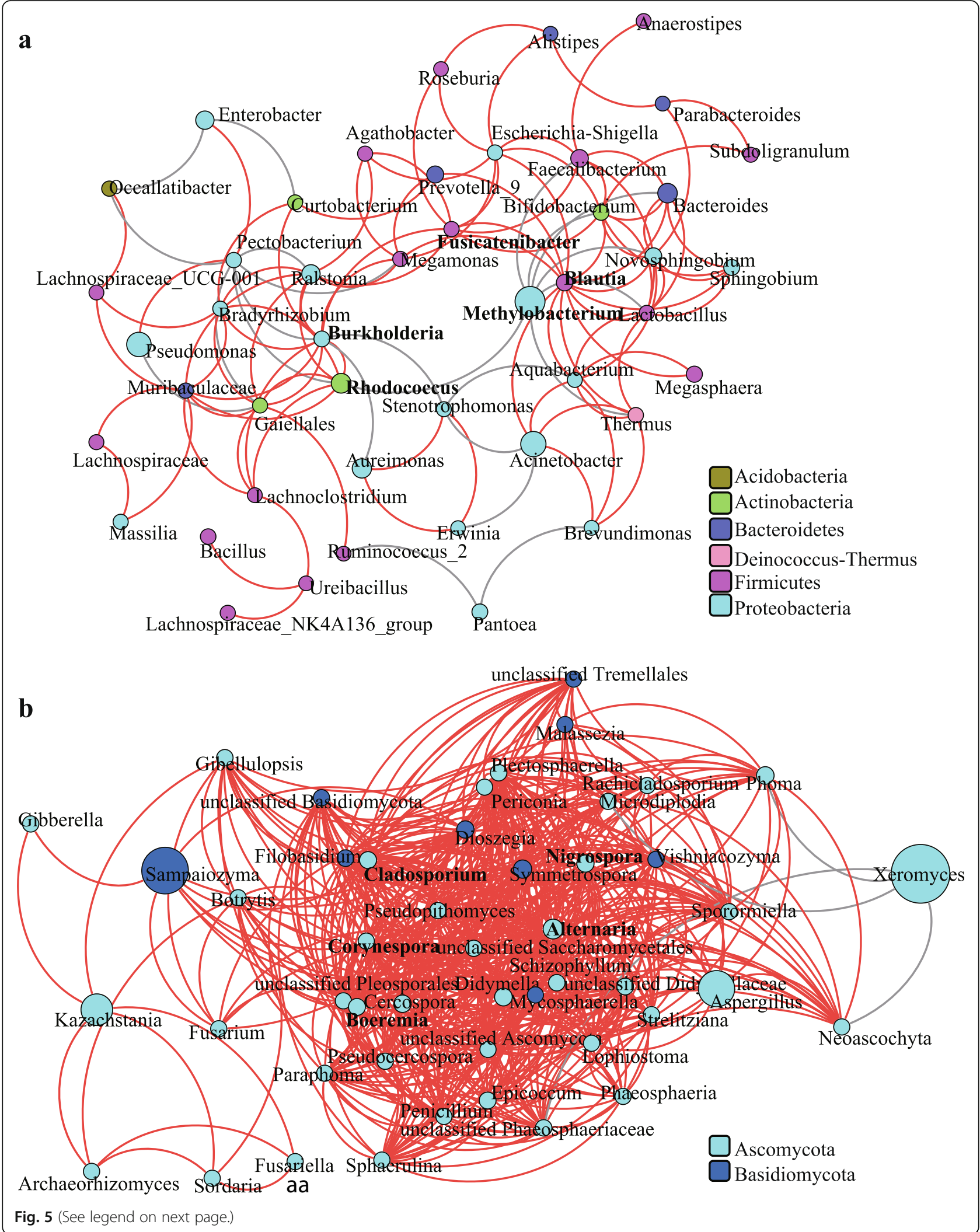


(See figure on previous page.)

Fig. 5 Network of co-occurring bacterial and fungal genera in tobacco leaves during the aging process based on correlation analysis. Networks a and b were constructed for bacterial and fungal genera, respectively, from five periods during the aging process. Only those genera with more than 10 sequences in at least 3 samples were included in the network analysis. A connection represented a statistically significant $(P<0.05)$ strong positive (Spearman's $r>0.6$ ) or negative (Spearman's $r<-0.6)$ correlation. The node colors indicate the phyla to which each genus belongs, and the node size is proportional to the abundance of taxa. The edges are colored based on interaction types; positive correlations are indicated in red and negative correlations in gray

and could be directly decomposed and utilized. The cellulase activity showed positive correlations with members of eight fungal genera, such as Strelitziana, Fusarium, Sphaerulina, Phaeosphaeria, Paraphoma, and Montagnula, and Enterobacter genus of bacterial phylum, but a negative correlation with Lachnospiraceae_ND3007_ group. The pectinase activity showed strong negative associations with most fungal genera such as Vishniacozyma, Golovinomyces, unclassified Tremellales, and unclassified Pleosporales, but a strong positive association with Subgroup_6 of the bacterial kingdom. These results indicated that microorganisms were closely related to biological enzymes during tobacco aging, and most of them had a promoting effect on enzyme activities.

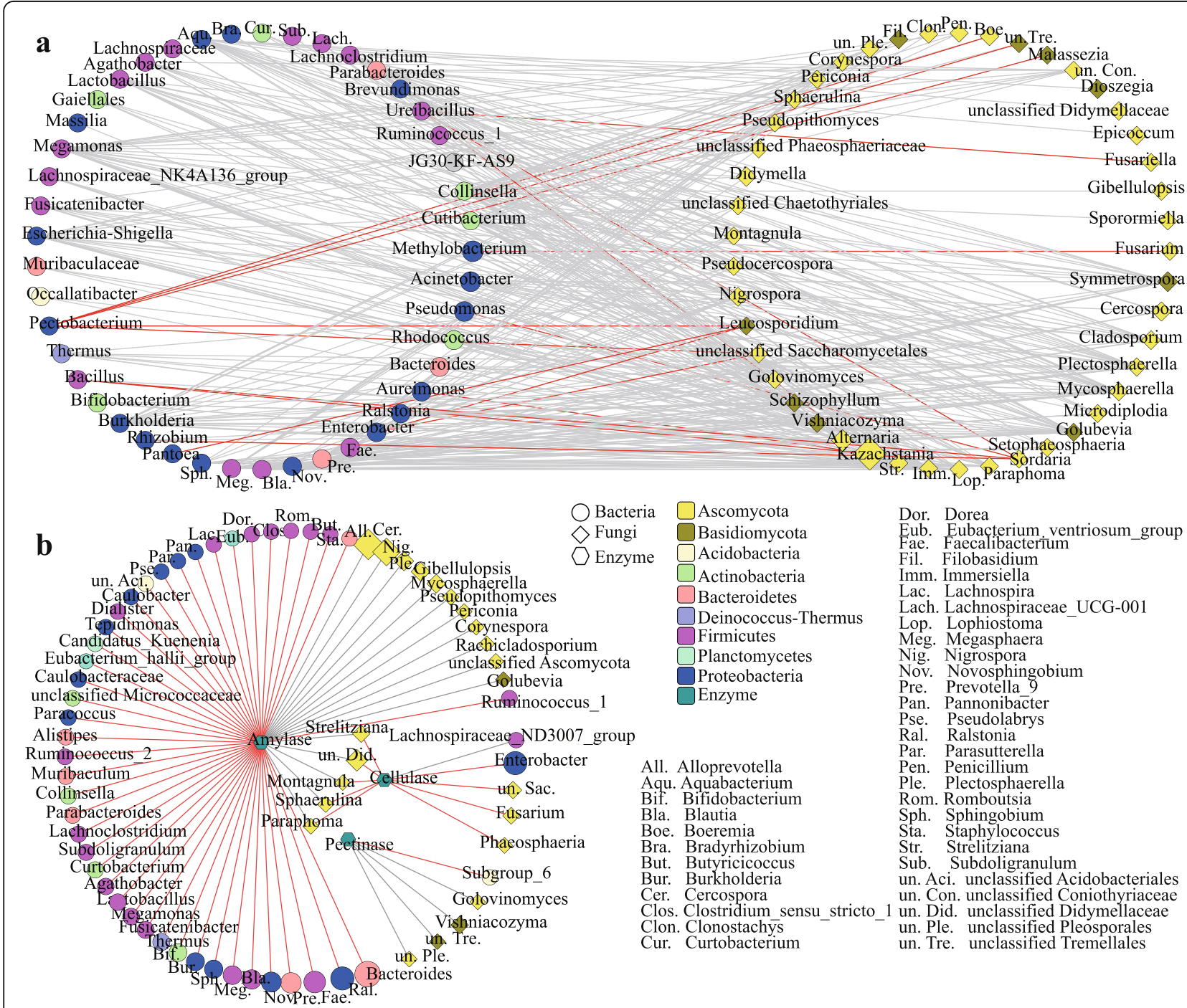

Fig. 6 Network of co-occurrence patterns between bacterial genus, fungal genus, and enzyme activities in tobacco leaves. Networks a and $\mathbf{b}$ were constructed based on the correlation analysis between bacteria and fungi and between microorganism (both bacteria and fungi) and enzyme activities, respectively. A connection represented a statistically significant $(P<0.05)$ strong positive (red, Spearman's $r>0.6)$ or negative (gray, Spearman's $r<-0.6)$ correlation. The node colors indicate the phyla to which each genus belongs, and the node size is proportional to the abundance of taxa 


\section{Discussion}

This study investigated the microbial community composition, diversity, and co-occurrence patterns in tobacco leaves during the aging process by Illumina HiSeq sequencing based on 16S rRNA and ITS genes. Proteobacteria, Firmicutes, Actinobacteria, Bacteroidetes, Acidobacteria, and Chloroflexi were abundant in the tobacco leaves during the aging process. Additionally, the most dominant phylum was Proteobacteria, which corroborated the results of Huang et al. (2010) and Su et al. (2011); however, this result was different from those of Wang et al. (2018) and Ye et al. (2017), who found that Firmicutes was the dominant phylum in flue-cured tobacco.

This study showed that $38.74-62.46 \%$ of predominant bacteria belonged to Sphingomonas, Methylobacterium, Pseudomonase, Acinetobacter, Enterobacter, Aureimonas, Bacteroides, and Rhodococcus. These genera were reported to be important contributors to nicotine degradation or the formation of representative flavor compounds (Zhang et al. 2020). For example, Ma et al. (2016) reported that a strain of Sphingomonas sp. isolated from tobacco leaves degraded chlorogenic acid into caffeic acid, shikimic acid, and 3,4-dihydroxybenzoic acid. Moreover, Enterobacter and Methylobacterium degraded sugars, organic acids, polyols, methanol, formate, and formaldehyde, which were present in the tobacco products (Rachman et al. 1997; Jourand et al. 2004). Pseudomonas is also interesting because of its ability to degrade nicotine, as demonstrated for Pseudomonas sp. Nic22 (Chen et al. 2008), Pseudomonas stutzeri ZCJ (Zhao et al. 2012), and Pseudomonas sp. HF-1 (Wang et al. 2013).

Ascomycota and Basidiomycota were two main fungal phyla in aged tobacco leaves, and Sampaiozyma, Aspergillus, and Xeromyces were dominant genera. However, this result did not agree with that of Zhang et al. (2020), who showed that Neophaeosphaeria and Cladosporium were the dominant genera in flue-cured tobacco leaves. Meng et al. (2010) reported that Aspergillus oryzae 112822 degraded nicotine to 2,3-dihydroxypyridine through the intermediates nornicotine, myosmine, $\mathrm{N}$ methylnicotinamide, and 2-hydroxy-N-methylnicotinamide. This finding indicated that the fungi also improved the quality of tobacco leaves. However, even more interestingly, most of these detected fungi (Aspergillus, Penicillium, Alternaria, and Cladosporium) caused mildew, which reduced the quality and use value of tobacco (Welty and Vickroy 1975).

However, environmental characteristics frequently affected the microbial community structure and function (Orland et al. 2019). In the tobacco aging process, the microenvironment can be altered by secondary metabolites produced during fermentation. For example, the levels of total organic acid and volatile compounds gradually increase, whereas the levels of nicotine, volatile alkali, total sugar, reducing sugar, and $\mathrm{pH}$ values decrease in tobacco leaves (Sun et al. 2011). Di Giacomo et al. (2007) reported that the structure and composition of the microbial community in Italian Toscano cigar changed significantly with the increase in temperature and $\mathrm{pH}$ during the fermentation process. This study found that the relative abundance of Proteobacteria decreased, whereas that of Actinobacteria and Bacteroidetes increased. The proportion of Ascomycota gradually increased during the first 18 months and then rapidly decreased, whereas Basidiomycota exhibited a completely opposite pattern. These results indicated that tobacco aging (or fermentation) is a microdynamic process dominated by microorganisms, whose composition and diversity may be affected by environmental conditions. In addition, this study found that the change in bacterial community structure in tobacco leaves was not significant in two different cities, but the change in fungal community structure was significant. This suggested that the fungi community might be more sensitive to environmental changes compared with bacteria.

Co-occurrence network analysis is useful for revealing common system-level properties of microbial communities in tobacco leaves during the aging process. Cooccurrence analysis of bacterial and fungal taxa from 10 tobacco samples in this study suggested strong withinand between-domain correlations among different groups of microorganisms in the tobacco leaves. In the bacterial community, the less-related interphylum genera had much higher co-occurrence ratios than the intraphylum genera in tobacco leaves. This finding was consistent with those reported by Zhang et al. (2020). However, genera from Ascomycota (intraphylum) showed a higher incidence of co-occurrence than other interphylum genera for the fungal community. These results indicated that microbial species might share niches and have synergistic or antagonistic relationships during tobacco aging (Barberán et al. 2012). However, the fungal network had a higher network density and clustering coefficient compared with the bacterial network, implying that the fungal network might have higher connectivity and stability than the bacterial network in the tobacco aging process. This result was consistent with those observed in the soil microbial community (Xiao et al. 2018; de Vries et al. 2018). The bacterium-fungus co-occurrence network showed that the incidence of negative correlation was significantly higher than that of positive correlation, indicating that most bacteria and fungi might be mutually exclusive in the tobacco aging ecosystem, which was similar to the bacterium-fungus interaction in the fermentation of $\mathrm{Pu}$ erh tea (Zhao et al. 2019a). In short, the interactions between microorganisms might be necessary to maintain the stability of the aging ecosystem during the aging process of tobacco leaves. 
Microorganisms are known to be the main manufacturers of enzymes in nature. For example, Aspergillus, Trichoderma, Alternaria, Fusarium, Penicillium, Rhizopus, and Cladosporium are common cellulolytic fungi that can convert native or derived cellulose to glucose (Jahangeer et al. 2005; Jin et al. 2012). Phanerochaete chrysosporium can degrade tobacco stalks by secreting cellulase (Su et al. 2016). The members of Enterobacteriaceae, Erwinia carotovora and Erwinia chrysanthemi, have been confirmed to degrade and utilize pectin (Abbott and Boraston 2008). During the aging process of tobacco leaves, microorganisms could synthesize and secrete enzymes to promote the degradation or transfer of complex macromolecular compounds. At present, many microorganisms inhabiting the surface of tobacco leaves have been confirmed to have strong enzymeproducing activities. For example, Dai et al. isolated two pectinase-producing strains, Bacillus subtilis and Alcaligenes faecalis, from the Zimbabwe tobacco strips (Dai et al. 2011). Guan et al. found a bacterial community, which consisted of Arthrobacter, Actinomycetes, Klebsiella, and two unknown bacteria, with the ability to decompose cellulose in aging flue-cured tobacco leaves (Guan et al. 2013). This study showed that the bacterial and fungal microbiomes were significantly associated with the activities of enzymes such as amylase, cellulase, and pectinase. This finding indicated that the microbial community was closely related to enzyme activity during tobacco aging, but the influence of microorganisms on enzyme activity depended on the type of microorganism and enzymes (Han et al. 1999). Residual enzymes in tobacco leaves are crucial in the early stage of aging, while microbial enzymes were important in the late stage of aging (Zhu et al. 2001). Furthermore, environment, time of aging, and enzyme structure affect the types and quantity of microorganisms (Larsen et al. 2019). However, this study only involved cellulase, pectinase, and amylase, with limited research indicators. Future studies should involve more enzymes and tobacco samples to verify the reliability and reproducibility of tobacco in different environments.

\section{Conclusions}

This study analyzed the microbial community dynamics and composition in tobacco leaves during the aging process by employing high-throughput sequencing techniques. It demonstrated that tobacco aging was a microdynamic process dominated by microorganisms, Shannon diversity, and Chao richness of bacterial communities gradually increased during the first 18 months, whereas those of the fungal community decreased. The relative abundance of Proteobacteria decreased, whereas that of Actinobacteria and Bacteroidetes increased. The proportion of Ascomycota gradually increased during the first 18 months and then rapidly decreased, whereas the proportion of Basidiomycota exhibited a completely opposite pattern. The change in bacterial community composition and dominant genera in tobacco leaves was not significant between GY and MT storerooms, but that in fungal community was significant. The network analysis revealed strong within- and between-domain correlations among different groups of microorganisms within the tobacco leaves. Fungal networks are more complex and compact than bacterial networks, and a strong negative correlation exists between bacteria and fungi. Moreover, the bacterial microbiome showed a strong positive association with amylase activity, while the fungal microbiome positively correlated with cellulase activity. These findings revealed the diversity, community composition, and co-occurrence pattern of tobacco bacterial and fungal communities during the aging process, thus providing a reference for tobacco microbial fermentation technology.

\section{Acknowledgements}

We would like to thank all the reviewers who participated in the review and MJEditor (www.mjeditor.com) for its linguistic assistance during the preparation of this manuscript.

\section{Authors' contributions}

Jiaxi Zhou and Xiao Zou conceived and designed the experiments. Jiaxi Zhou performed the experiments and analyzed the data. Xiao Zou acquired the funding and administered the project. Xiaomin Zhang and Jing Liu provided resources. Jiaxi Zhou, Lifei Yu, and Xiao Zou wrote, reviewed, and edited the manuscript. The author(s) read and approved the final manuscript.

\section{Funding}

This work was supported by the Science and Technology Project of Anshun Tobacco Company (201712), Science and Technology Project of Zunyi Tobacco Company (2018-02), and Science and Technology project of Shanghai Tobacco Group Co. LTD (K2016-1-018Z).

Availability of data and materials

The authors declare that all materials and data are available.

\section{Competing interest}

The authors declare that they have no conflict of interest.

Ethics approval and consent to participate

Not applicable.

Consent for publication

Not applicable.

\section{Author details}

${ }^{1}$ Institute of Fungal Resources, Department of Ecology, College of Life Sciences, Guizhou University, Guiyang, China. ${ }^{2}$ Guizhou Tobacco Company Zunyi Branch, Zunyi, China.

Received: 1 September 2020 Accepted: 6 January 2021

Published online: 01 February 2021

\section{References}

Abbott DW, Boraston AB (2008) Structural biology of pectin degradation by Enterobacteriaceae. Microbiol Mol Biol Rev 72(2):301-316

Adams RI, Miletto M, Taylor JW, Bruns TD (2013) Dispersal in microbes: fungi in indoor air are dominated by outdoor air and show dispersal limitation at short distances. ISME J 7:1262-1273

Assenov Y, Ramirez F, Schelhorn SE, Lengauer T, Albrecht M (2008) Computing topological parameters of biological networks. Bioinformatics 24(2):282-284 
Barberán A, Bates ST, Casamayor EO, Fierer N (2012) Using network analysis to explore co-occurrence patterns in soil microbial communities. ISME J 6(2): 343-351

Berry D, Widder S (2014) Deciphering microbial interactions and detecting keystone species with co-occurrence networks. Front Microbiol 5:219

Cao W, Xiong Y, Zhao D, Tan H, Qu J (2020) Bryophytes and the symbiotic microorganisms, the pioneers of vegetation restoration in karst rocky desertification areas in southwestern China. Appl Microbiol Biot 104(2):873-891

Chen C, Li X, Yang J, Gong X, Li B, Zhang K (2008) Isolation of nicotine-degrading bacterium Pseudomonas sp. Nic22, and its potential application in tobacco processing. Int Biodeter Biodegr 62(3):226-231

Chopyk J, Chattopadhyay S, Kulkarni P, Smyth EM, Hittle LE, Paulson JN, Pop M, Buehler SS, Clark PI, Mongodin EF, Sapkota AR (2017) Temporal variations in cigarette tobacco bacterial community composition and tobacco-specific nitrosamine content are influenced by brand and storage conditions. Front Microbiol 8(1):358

Dai T, Fan J, Zheng H, Bao K, Wang Y, Wang J (2011) Identification of pectindegrading strains isolated from tobacco strips and evaluation of its pectinase activity. Microbiol China 38(6):816-824

De Vries FT, Griffiths Rl, Bailey M, Craig H, Girlanda M, Gweon HS, Hallin S, Kaisermann A, Keith AM, Kretzschmar M, Lemanceau P, Lumini E, Mason KE, Oliver A, Ostle N, Prosser Jl, Thion C, Thomson B, Bardgett RD (2018) Soil bacterial networks are less stable under drought than fungal networks. Nat Commun 9:3033

Di Giacomo M, Paolino M, Silvestro D, Vigliotta G, Imperi F, Visca P, Alifano P, Parente D (2007) Microbial community structure and dynamics of dark firecured tobacco fermentation. Appl Environ Microbiol 73(3):825-837

Edgar RC (2013) UPARSE: highly accurate OTU sequences from microbial amplicon reads. Nat Methods 10(10):996-998

Gonzalez AM, Dalsgaard B, Olesen JM (2010) Centrality measures and the importance of generalist species in pollination networks. Ecol Complex 7(1): 36-43

Guan L, Li T, Chen Z, Zhou Y, Tang C, Qu Y, Li P (2013) Isolation and characterization of bacterial community with cellulose-decomposition ability from aging flue-cured tobacco leaves. Chinese Tobacco Sci 34(6):103-107

Han J, Zhu D, Yang S, Ye B (1999) Analysis of enzyme activity and relevant chemical components of flue-cured tobacco in different stages of aging. Chinese Tobacco Sci 1(1):1-2

Huang J, Yang J, Duan Y, Gu W, Gong X, Zhe W, Su C, Zhang K (2010) Bacterial diversities on unaged and aging flue-cured tobacco leaves estimated by $16 \mathrm{~S}$ rRNA sequence analysis. Appl Microbiol Biot 88(2):553-562

Jahangeer S, Khan N, Jahangeer S, Sohail M, Khan ASA (2005) Screening and characterization of fungal cellulases isolated from the native environmental source. Pak J Bot 37(3):739-748

Jin R, Liao H, Liu X, Zheng M, Xiong X, Liu X, Zhang L, Zhu Y (2012) Identification and characterization of a fungal strain with lignin and cellulose hydrolysis activities. Afr J Microbiol Res 6(36):6545-6550

Jourand P, Giraud E, Bena G, Sy A, Willems A, Gillis M, Dreyfus B, de Lajudie P (2004) Methylobacterium nodulans sp. nov., for a group of aerobic, facultatively methylotrophic, legume root-nodule-forming and nitrogenfixing bacteria. Int J Syst Evol Micr 54(6):2269-2273

Kinkel LL (1997) Microbial population dynamics on leaves. Annu Rev Phytopathol 35(1):327-347

Köljalg U, Nilsson RH, Abarenkov K, Tedersoo L, Taylor AFS, Bahram M, Bates ST, Bruns TD, Bengtsson-Palme J, Callaghan TM, Douglas B, Drenkhan T, Eberhardt U, Dueñas M, Grebenc T, Griffith GW, Hartmann M, Kirk PM, Kohout $P$, Larsson E, Lindahl BD, Lücking R, Martín MP, Matheny PB, Nguyen $\mathrm{NH}$, Niskanen T, Oja J, Peay KG, Peintner U, Peterson M, Põldmaa K, Saag L, Saar I, Schüßler A, Scott JA, Senés C, Smith ME, Suija A, Taylor DL, Telleria MT, Weiss M, Larsson KH (2013) Towards a unified paradigm for sequence-based identification of fungi. Mol Ecol 22(21):5271-5277

Larsen N, Bussolo de Souza C, Krych L, Barbosa Cahú T, Wiese M, Kot W, Hansen KM, Blennow A, Venema K, Jespersen L (2019) Potential of pectins to beneficially modulate the gut microbiota depends on their structural properties. Front. Microbiol 10:223

Larsson L, Szponar B, Ridha B, Pehrson C, Dutkiewicz J, Krysińska-Traczyk E, Sitkowska J (2008) Identification of bacterial and fungal components in tobacco and tobacco smoke. Tob Induc Dis 4(4):1-8

Law AD, Fisher C, Jack A, Moe LA (2016) Tobacco, microbes, and carcinogens: correlation between tobacco cure conditions, tobacco-specific nitrosamine content, and cured leaf microbial community. Microb Ecol 72(1):120-129
Lian B, Wang NY, Deng J (1998) Analysis of tobacco mildew and its prevention and cure. Guizhou Sci 16:124-127

Liu J, Ma G, Chen T, Hou Y, Yang S, Zhang K, Yang J (2015) Nicotine-degrading microorganisms and their potential applications. Appl Microbiol Biot 99(9): 3775-3785

Ma Y, Wang X, Nie X, Zhang Z, Yang Z, Nie C, Tang H (2016) Microbial degradation of chlorogenic acid by a Sphingomonas sp. strain. Appl Biochem Biotech 179(8):1381-1392

Magoč T, Salzberg SL (2011) FLASH: fast length adjustment of short reads to improve genome assemblies. Bioinformatics 27(21):2957-2963

Maldonado-Robledo G, Rodriguez-Bustamante E, Sanchez-Contreras A, RodriguezSanoja R, Sanchez S (2003) Production of tobacco aroma from lutein. Specific role of the microorganisms involved in the process. Appl Microbiol Biot 62(5-6):484-488

Malhotra R, Noorwez SM, Satyanarayana T (2000) Production and partial characterization of thermostable and calcium-independent a-amylase of an extreme thermophile Bacillus thermooleovorans NP54. Lett Appl Microbiol 31(5):378-384

Meng X, Lu L, Gu G, Xiao M (2010) A novel pathway for nicotine degradation by Aspergillus oryzae 112822 isolated from tobacco leaves. Res Microbiol 161(7): 626-633

Minjares-Carranco A, Trejo-Aguilar BA, Aguilar G, Viniegra-González G (1997) Physiological comparison between pectinase-producing mutants of Aspergillus niger adapted either to solid-state fermentation or submerged fermentation. Enzyme Microb Tech 21:25-31

Orland C, Emilson EJS, Basiliko N, Mykytczuk NCS, Gunn JM, Tanentzap AJ (2019) Microbiome functioning depends on individual and interactive effects of the environment and community structure. ISME J 13(1):1-11

Rachman MA, Furutani Y, Nakashimada Y, Kakizono T, Nishio N (1997) Enhanced hydrogen production in altered mixed acid fermentation of glucose by Enterobacter aerogenes. J Ferment Bioeng 83(4):358-363

Rognes T, Flouri T, Nichols B, Quince C, Mahé F (2016) VSEARCH: a versatile open source tool for metagenomics. Peer J 4:e2584

Rui J, Li J, Zhang S, Yan X, Wang Y, Li X (2015) The core populations and cooccurrence patterns of prokaryotic communities in household biogas digesters. Biotechnol Biofuels 8:158

Shannon P, Markiel A, Ozier O, Baliga NS, Wang JT, Ramage D, Amin N, Schwikowski B, Ideker T (2003) Cytoscape: a software environment for integrated models of biomolecular interaction networks. Genome Res 13(11): 2498-2504

Shetty SA, Hugenholtz F, Lahti L, Smidt H, de Vos WM (2017) Intestinal microbiome landscaping: insight in community assemblage and implications for microbial modulation strategies. FEMS Microbiol Rev 41(2):182-199

Sporns O, Honey CJ, Kotter R (2007) Identification and classification of hubs in brain networks. PLOS ONE 2(10):e1049

Su C, Gu W, Zhe W, Zhang K, Duan Y, Yang J (2011) Diversity and phylogeny of bacteria on Zimbabwe tobacco leaves estimated by 165 rRNA sequence analysis. Appl Microbiol Biot 92(5):1033-1044

Su Y, Xian H, Shi S, Zhang C, Manik SMN, Mao J, Zhang G, Liao W, Wang Q, Liu H (2016) Biodegradation of lignin and nicotine with white rot fungi for the delignification and detoxification of tobacco stalk. BMC Biotechnol 16(1):81

Sun J, He J, Wu F, Tu S, Yan T, Si H, Xia H (2011) Comparative analysis on chemical components and sensory quality of aging flue-cured tobacco from four main tobacco areas of China. Agr Sci China 10(8):1222-1231

Vigliotta G, Di Giacomo M, Carata E, Massardo DR, Tredici SM, Silvestro D, Paolino M, Pontieri P, Del Giudice L, Parente D, Alifano P (2007) Nitrite metabolism in Debaryomyces hansenii TOB-Y7, a yeast strain involved in tobacco fermentation. Appl Microbiol Biot 75(3):633-645

Villemur R, Lacasse M, Morin A (2009) Monitoring the bacterial and fungal biota of eleven tobacco grades stored at three different locations. Beiträge zur Tabakforschung International/Contributions to Tobacco Research 23(6):368-376

Wang F, Zhao H, Xiang H, Wu L, Men X, Qi C, Chen G, Zhang H, Wang Y, Xian M (2018) Species diversity and functional prediction of surface bacterial communities on aging flue-cured tobaccos. Curr Microbiol 75:1306-1315

Wang T, Qiu H, Chen H, An Y, Ding L (2008) Applying 3,5-dinitrosalicylic acid method to measured pectinase activity. Food Mach 24(3):96-99

Wang X, Tang L, Yao Y, Wang H, Min H, Lu Z (2013) Bioremediation of the tobacco waste-contaminated soil by Pseudomonas sp. HF-1: nicotine degradation and microbial community analysis. Appl Microbiol Biot 97(13): $6077-6088$ 
Welty RE, Vickroy DG (1975) Evaluations of cigarettes made with mold-damaged and nondamaged flue-cured tobacco. Beiträge zur Tabakforschung/ Contributions to Tobacco Research 8(2):102-106

Xiao X, Liang Y, Zhou S, Zhuang S, Sun B (2018) Fungal community reveals less dispersal limitation and potentially more connected network than that of bacteria in bamboo forest soils. Mol Ecol 27(2):550-563

Xu N, Tan G, Wang H, Gai X (2016) Effect of biochar additions to soil on nitrogen leaching, microbial biomass and bacterial community structure. Eur J Soil Biol $74: 1-8$

Ye J, Yan J, Zhang Z, Yang Z, Liu X, Zhou H, Wang G, Hao H, Ma K, Ma Y, Mao D, Yang $X$ (2017) The effects of threshing and redrying on bacterial communities that inhabit the surface of tobacco leaves. Appl Microbiol Biot 101(10):4279-4287

Zhang Q, Geng Z, Li D, Ding Z (2020) Characterization and discrimination of microbial community and co-occurrence patterns in fresh and strong flavor style flue-cured tobacco leaves. MicrobiologyOpen 9:e965

Zhao D, Huang R, Zeng J, Yu Z, Liu P, Cheng S, Wu Q (2014) Pyrosequencing analysis of bacterial community and assembly in activated sludge samples from different geographic regions in China. Appl Microbiol Biot 98(21):9119-9128

Zhao L, Zhu C, Gao Y, Wang C, Li X, Shu M, Shi Y, Zhong W (2012) Nicotine degradation enhancement by Pseudomonas stutzeri ZCJ during aging process of tobacco leaves. World J Microbiol Biotechnol 28(5):2077-2086

Zhao M, Su X, Nian B, Chen L, Zhang D, Duan S, Wang L, Shi X, Jiang B, Jiang W, Lv C, Wang D, Shi Y, Xiao Y, Wu J, Pan Y, Ma Y (2019a) Integrated metaomics approaches to understand the microbiome of spontaneous fermentation of traditional Chinese pu-erh tea. mSystems 4:e00680-19

Zhao M, Wang B, Li F, Qiu L, Li F, Wang S, Cui J (2007) Analysis of bacterial communities on aging flue-cured tobacco leaves by $16 \mathrm{~S}$ rDNA PCR-DGGE technology. Appl Microbiol Biot 73(6):1435-1440

Zhao Z, He J, Geisen S, Han L, Wang J, Shen J, Wei W, Fang Y, Li P, Zhang L (2019b) Protist communities are more sensitive to nitrogen fertilization than other microorganisms in diverse agricultural soils. Microbiome 7(1):33

Zhou J, Yu L, Zhang J, Zhang X, Xue Y, Liu J, Zou X (2020) Characterization of the core microbiome in tobacco leaves during aging. MicrobiologyOpen 9:e984

Zhu D, Chen R, Chen Z, Zhou Y, Han J, Jin B, Song R, Yu J (2001) The relationship between microorganisms and enzyme activities in flue-cured tobacco during aging and fermentation. Acta Tabacaria Sinica 7(2):26-30

\section{Publisher's Note}

Springer Nature remains neutral with regard to jurisdictional claims in published maps and institutional affiliations.

Ready to submit your research? Choose BMC and benefit from:

- fast, convenient online submission

- thorough peer review by experienced researchers in your field

- rapid publication on acceptance

- support for research data, including large and complex data types

- gold Open Access which fosters wider collaboration and increased citations

- maximum visibility for your research: over $100 \mathrm{M}$ website views per year

At $\mathrm{BMC}$, research is always in progress.

Learn more biomedcentral.com/submissions 